\title{
Distalising upper first permanent molars
}

\author{
Is the Jones Jig an effective method of distalising upper first permanent molars?
}

Paul LD, O'Brien KD, Mandall NA. Upper removable appliance or Jones Jig for distalising first molars? A randomised clinical trial. Orthod Craniofac Res 2002; 5:238-242

Design This was a randomised controlled trial (RCT).

Intervention The RCT examined use of the Jones jig or an upper removable appliance.

Outcome measure Changes in the position of the upper-first permanent molar were measured, in terms of distal movement, distal tipping and distopalatal rotation (molar straightening). Also recorded were mesial movement of the upper-first premolars (loss of anchorage) and any reported discomfort.

Results There were no statistically significant differences between the two treatment methods for any of the outcome measures (see Table 1). Distal movement obtained by both appliances was approximately $1 \mathrm{~mm}$.

Table 1. Amount and type of tooth movement produced by the upper removable appliance (URA) and the Jones Jig.

\begin{tabular}{|c|c|c|c|}
\hline \multirow[b]{2}{*}{ Tooth movement } & \multicolumn{2}{|c|}{ Appliance (mean (SD)) } & \multirow[b]{2}{*}{$P$} \\
\hline & Jones Jig & URA & \\
\hline Tipping (degrees) & $4.56(3.31)$ & $3.18(5.12)$ & 0.47 \\
\hline Mesial premolar movement (mm) & $0.18(2.30)$ & $0.18(2.26)$ & 0.70 \\
\hline Rotation (degrees) & $5.16(4.31)$ & $2.92(3.63)$ & 0.19 \\
\hline Distal molar movement (mm) & $1.17(1.94)$ & $1.30(1.34)$ & 0.85 \\
\hline $\begin{array}{l}\text { Reported discomfort } \\
\quad(1, \text { no pain; } 9 \text {, severe pain })\end{array}$ & $2.39(1.0)$ & $2.80(1.54)$ & 0.47 \\
\hline
\end{tabular}

Conclusions The amount of distal movement obtained with both appliances was small and no differences were shown in the amount of molar tooth movement. It is suggested that there is no advantage in using the Jones Jig as a noncompliance appliance.
Address for correspondence: NA Mandall, Orthodontic Unit, Department of Dental Medicine and Surgery, University Dental Hospital of Manchester, Higher Cambridge Street, Manchester M15 6FH, UK. E-mail: nicky@fs1.den.man.ac.uk

\section{Commentary}

Appliances may be chosen by clinicians either for their perceived effectiveness or compliance requirements. The clinical evidence to support these choices is needed and would be beneficial in dental practice. Therefore, this study investigated the effectiveness of a removable and a fixed Jones Jig appliance to orthodontically distalise maxillary molars.

The sample size was determined that would allow detection of a difference of $3 \mathrm{~mm}$ tooth movement, which represents a relevant clinical change. The sample size was not calculated, however, to detect a difference for the other two parameters assessed. This raises questions over the validity of the results for mesial movement and rotation. The method of randomisation of patients is unclear, especially for randomised blocks of 12 when there were only 12 and 11 patients in each group. The classification of this as a true RCT is questionable.

The control of confounding variables such as treatment protocols — including appliance wear, frequency and amount of adjustments - and sample variability is an issue in trials. In this study, an important confounder is the stage of dental development, and the wide age range of individuals at the start of treatment (10 and 16 years) encompasses both patients who do and do not yet have erupted second permanent molars. The distalisation of a first permanent molar against an erupted second permanent molar should not be compared with distalisation without an erupted second permanent molar. Although one might try, most clinicians would not expect to achieve any significant distalisation of maxillary first permanent molars with a removable appliance when the maxillary second permanent molars are erupted. Excluding subjects who have erupted second permanent molars would enable a better comparison of the appliances.

Another possible confounding variable is the type of initial malocclusion that the patients had, that is, deep bites, open bites or associated habits.

Unfortunately, the characteristics of the groups are not sufficiently delineated to assign the lack of a difference solely to the appliance. Whether the five clinicians used same treatment protocols and instructions for patients is not stated, which could influence the outcomes. Although the outcomes were assessed by blinded examiners, all the patients who entered the study were not properly accounted for at the end of the study. The authors should have statistically assessed the entire group of 27 patients and then statistically accounted for the four patients who were not included in the final study. It was incorrect statistically to eliminate the four and complete the analysis on the 23 patients.

In summary, problems with the sample and methodology do not allow for a valid assessment of the effectiveness of these appliances.

\section{Kathy Russell}

Division of Orthodontics, Faculty of Dentistry, Dalhousie

University, Halifax, Nova Scotia, Canada

Evidence-Based Dentistry (2004) 5, 77.

doi:10.1038/sj.ebd.6400274 